\title{
Editorial
}

\section{Functional Polymers for Biointerface Engineering}

\author{
Qiang Wei, ${ }^{1}$ Jie He, ${ }^{2}$ Weifeng Zhao, ${ }^{3}$ and Yin $\mathrm{Chen}^{4}$ \\ ${ }^{1}$ Max Planck Institute for Medical Research, Stuttgart, Germany \\ ${ }^{2}$ University of Connecticut, Storrs, CT, USA \\ ${ }^{3}$ Sichuan University, Chengdu, China \\ ${ }^{4}$ The Hong Kong University of Science and Technology, Hong Kong, China \\ Correspondence should be addressed to Qiang Wei; wei@is.mpg.de, Jie He; jie.he@uconn.edu, \\ Weifeng Zhao; weifeng@kth.se, and Yin Chen; yinchen@ust.hk
}

Received 2 January 2017; Accepted 4 January 2017; Published 28 February 2017

Copyright (c) 2017 Qiang Wei et al. This is an open access article distributed under the Creative Commons Attribution License, which permits unrestricted use, distribution, and reproduction in any medium, provided the original work is properly cited.

Biomaterials and biomedical devices can induce a set of adverse biological responses upon contact with body fluids and/or implantation into living organs, as a result of the formation of biointerfaces between biomolecules and surfaces. Functional polymers have been of great interest because of their promising properties for improving the biocompatibility of the materials and mimicking the biological systems to stimulate/interact with cells. Given the great potential of polymers in solving problems at biointerfaces and the growing demands in their biomedical applications, we launched this special issue, which includes seven research articles, to illustrate the applications of functional polymers in engineering biointerfaces between biological systems and polymeric materials and preparing novel biomaterials with desired surface characters.

Surface modification of solid materials is a key to generating artificial biointerface. When bioinert and/or bioactive polymers are immobilized on the surface of biomaterials and biomedical devices via modern fabrication technologies, newly resulting biointerfaces exhibit versatile biological effects, including antifouling, anticoagulation, specific adhesion, and tissue regeneration [1]. The strategies of generating polymeric surfaces include "grafting from," "grafting to," and "blending" techniques. The article "Bottom-Up Fabrication of PEG Brush on Poly(dimethylsiloxane) for Antifouling Surface Construction" and the article "Constructing Functional Ionic Membrane Surface by Electrochemically Mediated Atom Transfer Radical Polymerization" show the "grafting from" technique via surface-initiated reversible addition-fragmentation chain transfer (SI-RAFT) polymerization and surface-initiated atom transfer radical polymerization (SI-ATRP), respectively. The "grafting to" technique was utilized in the article "Use of Oxalic-Acid-Modified Stellerite for Improving the Filter Capability of $\mathrm{PM}_{2.5}$ of Paper Composed of Bamboo Residues" to immobilize oxalic acid and cationic starch on the surface of stellerites and to further stabilize the modified stellerites on the paper sheets. As an alternative way, the surface of ultrafiltration membrane was modified by simply blending polyurethane in the article "Bio-Inspired Polyethersulfone Membrane Design via Blending with Functional Polyurethane." As such, the biocompatibility, hemocompatibility, and specific filtration ability of the materials were improved, which were highly required to meet the growing demands of the biomedical applications. Besides the traditional specific surface coatings shown above, materials-independent universal polymer coatings are increasing needed because of the fast development and diversification in materials science. The general rules were proposed to develop this type of coatings [2]: (1) there must be appropriate interactions between the coating polymers and the substrates; (2) intracoating crosslinking is required to stabilize the polymer coatings; (3) the coatings should present available functions or reactive functionalities for further functionalization.

The scale size of the polymeric materials also results in versatile effects at biointerfaces, for example, benefiting the rapid responses to external stimuli [3]. Thanks to the rapid development of nanotechnology, the polymers in 
microscale and nanoscale have been extensively studied. The advantages of nanomaterials/macromaterials are often due to the high surface/volume ratios. Generally, the construction of a nanoscale or microscale system requires synthesis or fabrication of scalable polymers, suitable devices, and so on. For example, the article "Influence of Sodium Alginate on Hypoglycemic Activity of Metformin Hydrochloride in the Microspheres Obtained by the Spray Drying" employed spray drying approach towards fabricating soft microgels for metformin hydrochloride delivery, which could improve the lipid profile in human body with no risk of hypoglycemia. By incorporating $\mathrm{Fe}_{3} \mathrm{O}_{4}$ nanoparticles into polysaccharide hydrogels, the soft drug controlled delivery system could be achieved by magnetic field stimuli [4]. For repairing hard tissues, the article "The Influence of Irradiation and Accelerated Aging on the Mechanical and Tribological Properties of the Graphene Oxide/Ultra-High-MolecularWeight Polyethylene Nanocomposites" introduces nanosize graphene oxide into widely used ultra-high-molecularweight polyethylene for improvement of mechanical properties of artificial joints. Generally, high repairing efficiency, low toxicity, and good tolerance should be taken into consideration during use of nanoscale/microscale polymers in biomedical applications [5].

Natural biopolymers are macromolecules derived from biological systems, such as DNA, protein, and polysaccharide. They have been extensively used as biomaterials for drug delivery, disease diagnosis, wound dressing, and tissue engineering as a result of their good biocompatibility and programmable biodegradability [6] or even directly as biospecific stimulants for tuning cell behaviors via the surface receptors of cells and tissues. In the article "Fish Collagen Promotes the Expression of Genes Related to Osteoblastic Activity," fish collagen was demonstrated to promote the differentiation and maturation of a preosteoblast cell line (MC3T3-E1).

In summary, this special issue bridges the polymer science and the biointerface engineering. We sincerely hope that the readers enjoy reading the presented original research work in this special issue and they can get inspired for their future studies.

\section{Acknowledgments}

We appreciate the great effort and the kind contributions from the authors, reviewers, and the editorial staff members to this special issue.

\footnotetext{
Qiang Wei $\mathrm{Jie} \mathrm{He}$

Weifeng Zhao

Yin Chen
}

[2] Q. Wei and R. Haag, "Universal polymer coatings and their representative biomedical applications," Materials Horizons, vol. 2, no. 6, pp. 567-577, 2015.

[3] W. Zhao, R. W. N. Nugroho, K. Odelius, U. Edlund, C. Zhao, and A.-C. Albertsson, "In situ cross-linking of stimuli-responsive hemicellulose microgels during spray drying," ACS Applied Materials \& Interfaces, vol. 7, no. 7, pp. 4202-4215, 2015.

[4] W. Zhao, K. Odelius, U. Edlund, C. Zhao, and A.-C. Albertsson, "In situ synthesis of magnetic field-responsive hemicellulose hydrogels for drug delivery," Biomacromolecules, vol. 16, no. 8, pp. 2522-2528, 2015.

[5] H. Malhaire, J.-C. Gimel, E. Roger, J.-P. Benoît, and F. Lagarce, "How to design the surface of peptide-loaded nanoparticles for efficient oral bioavailability?" Advanced Drug Delivery Reviews, vol. 106, pp. 320-336, 2016.

[6] S. Van Vlierberghe, P. Dubruel, and E. Schacht, "Biopolymerbased hydrogels as scaffolds for tissue engineering applications: a review," Biomacromolecules, vol. 12, no. 5, pp. 1387-1408, 2011.

\section{References}

[1] Q. Wei, T. Becherer, S. Angioletti-Uberti et al., "Protein interactions with polymer coatings and biomaterials," Angewandte Chemie-International Edition, vol. 53, no. 31, pp. 8004-8031, 2014. 

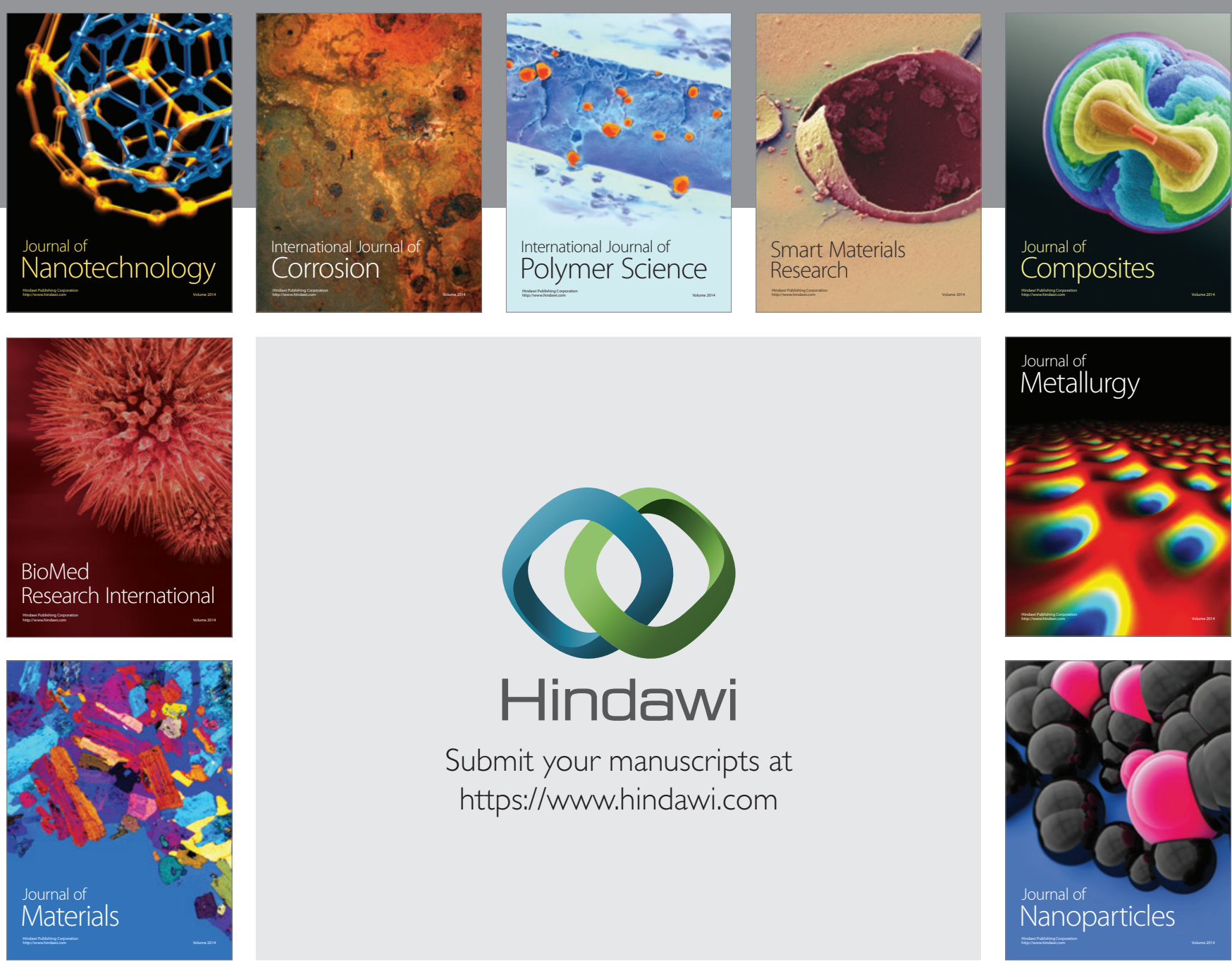

\section{Hindawi}

Submit your manuscripts at

https://www.hindawi.com

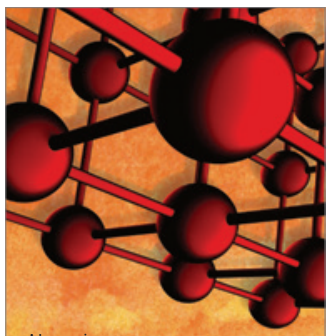

Materials Science and Engineering
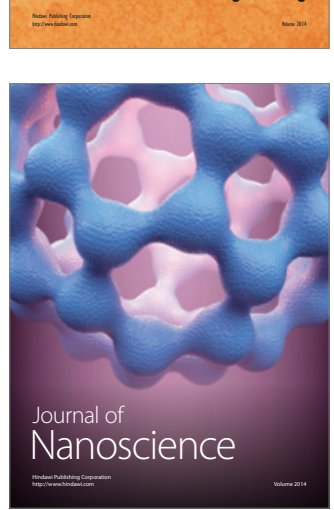
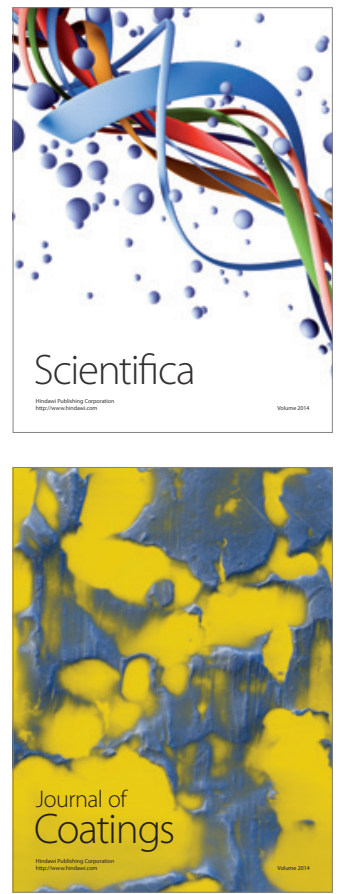
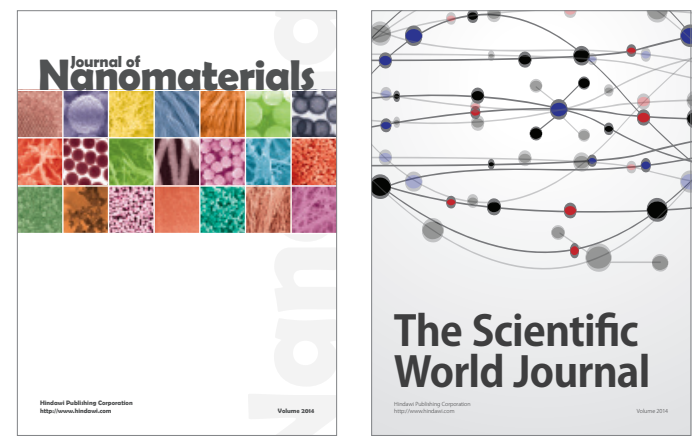

The Scientific World Journal
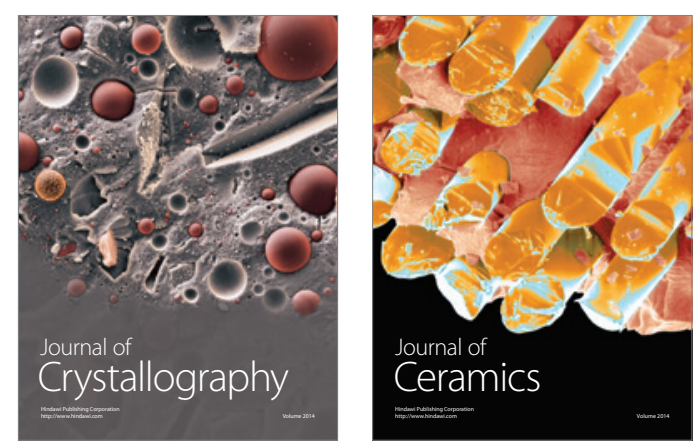
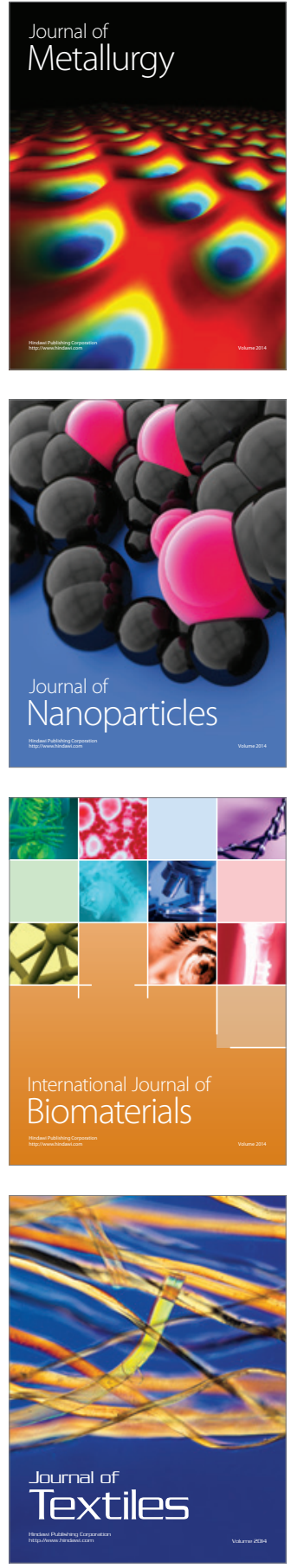